\title{
Femtosecond and Attosecond Spectroscopy in the XUV regime
}

\author{
Arvinder S. Sandhu and Xiao-Min Tong
}

\begin{abstract}
Attosecond-duration, fully coherent, extremeultraviolet (XUV) photon bursts obtained through laser highharmonic generation have opened up new possibilities in the study of atomic and molecular dynamics. We discuss experiments elucidating some of the interesting energy redistribution mechanisms that follow the interaction of a highenergy photon with a molecule. Crucial role of synchronized, strong-field, near-infrared laser pulses in XUV pump-probe spectroscopy is highlighted. We demonstrate that near-infrared (IR) pulses can in fact be used to modify the atomic structure and control the electronic dynamics on attosecond timescales. Our measurements show that the Gouy phase slip in the interaction region plays a significant role in these attosecond experiments. We perform precision measurement of interferences between strong field-induced Floquet channels to extract the intensity and phase dependence of photo-ionization dynamics. Applications of emerging table-top ultrafast XUV sources in study of core electron dynamics are also discussed.
\end{abstract}

Index Terms - Attosecond, Femtosecond, High-harmonics, Strong field dynamics, XUV spectroscopy.

\section{INTRODUCTION}

$\mathrm{U}$ ltrafast atomic and molecular science has undergone quite a revolution in the last few years. One of the major changes has been brought about by the arrival of ultrafast table-top XUV sources in the form of attosecond-duration, high-frequency, coherent light pulses. The birth of a new subfield, being termed as 'attosecond science', has been made possible by advances in laser technology, detection methods and theoretical calculations.[1] The excitement in this field arises from the possibility of observation and control at the level of electrons[2-7], which typically undergo dynamics on the timescale of few-hundred attoseconds.

The phenomenon underlying ultrashort XUV pulses is the extreme non-linear interaction of intense near-infrared (IR) laser pulses $\left(>10^{14} \mathrm{~W} \mathrm{~cm}^{-2}\right)$ with atoms, which leads to the generation of laser high-harmonics extending upto 100's eV $[8,9]$. Time-resolved experiments with these laser-generated

Manuscript received December 15, 2010. This work was supported by the National Science Foundation, USA (Grant PHY-0955274).

A. S. Sandhu is with the Department of Physics and College of Optical Sciences, University of Arizona, Tucson, AZ 85737 USA (corresponding author; phone: 520-621-6786; fax: 520-621-4721; e-mail: sandhu@ physics.arizona.edu).

X.-M. Tong is with the Center for Computational Sciences, University of Tsukuba, Ibaraki 305-8573, Japan (e-mail: tong@ims.tsukuba.ac.jp). ultrashort XUV pulses provide new insights into electronic processes in atoms, molecules, and surfaces [1-3, 5-7, 10-20].

Strictly speaking, apart from attosecond XUV pulses, the implementation of ultrafast XUV spectroscopy also requires precisely synchronized strong field IR laser pulses. In fact, many new experimental possibilities have emerged from this marriage between conventional strong-field (IR) and weak field (XUV) spectroscopic techniques. We refer to this entire class of experiments as ultrafast XUV+IR spectroscopy.

In the framework of XUV+IR spectroscopy, we will focus on the developments along two main directions. One is the utilization of XUV pulses for initiation of ultrafast chemical dynamics through inner-valence/multi-electron excitations. The resulting femtosecond electronic and nuclear processes in such experiments are probed using time-delayed IR pulses. Other direction represents the use of attosecond duration XUV pulse trains to study and control the transient, strong field induced modification of the electronic structure of atoms. In these experiments, the XUV and IR fields are simultaneously present and their relative delay is precisely controlled on attosecond timescale.

A brief outline of the paper is as follows. In section II we show that ultrashort XUV excitation is a precursor of novel excited-state dynamics. The role of IR pulses in pump-probe measurement of XUV excited electronic and nuclear wavepackets is investigated in section III. Experimental methods are discussed in section IV. Section V and VI focus on the attosecond measurements of XUV+IR ionization dynamics. The significance of Gouy phase slip and advantages of spatial imaging of focal volume are demonstrated. We use Floquet formalism to interpret the behavior of atomic structures in strong fields. We show that ion-yield oscillations encode the IR field strength dependence of transition matrix elements. Lastly, we discuss the new experimental possibilities in study of multi-electron and core electron dynamics.

\section{Ultrashort XUV PULSE INITIATED DYNAMICS}

Most physical and chemical phenomena occur through photo-excitation and subsequent evolution of electronic and nuclear wavepackets[21]. Ultrashort light pulses in infrared (IR), visible and ultraviolet (UV) have been extensively used to uncover the nature and dynamics of excited molecular states[22]. However, most time-resolved studies of 


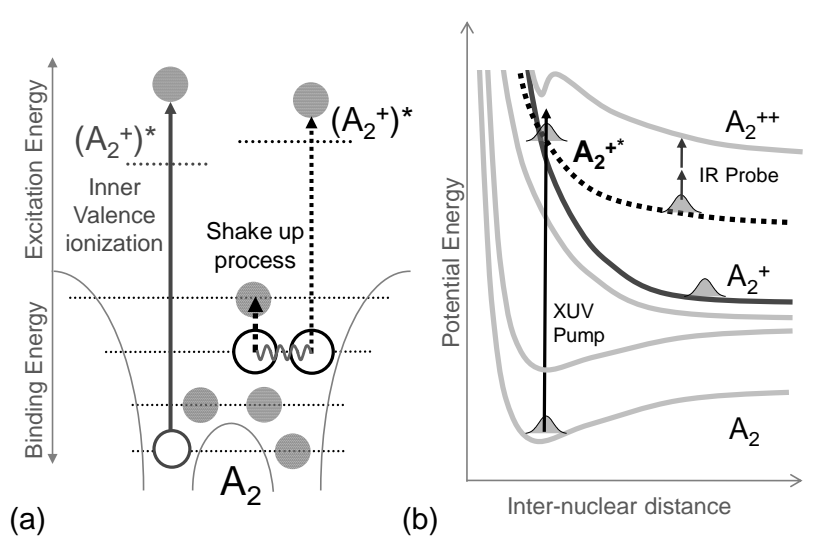

Fig. 1 Inner-shell photoionization and correlated two-electron shakeup photoionization (b) either case leads to formation of highly-excited molecular ions which undergo fast fragmentation along multiple pathways.

wavepacket dynamics have been limited to low-lying excited states, only few eV above the ground state. In contrast, many common processes in nature (e.g., solar irradiation of atmospheric molecules) lead to formation of excited molecules high above the ground state $(>20 \mathrm{eV})$.

Figure 1(a) illustrates the XUV photon initiated inner-shell ionization (solid line) and two-electron shake-up processes (dotted line) in a diatomic molecule generically labeled as $\mathrm{A}_{2}$. Such processes form highly-excited molecules in the multiple continua above the single or even double ionization threshold. The complex energy relaxation paths often involve fragmentation along steep potential energy surfaces as those depicted in figure 1(b). The evolution of such highly-excited states presents an interesting realm which involves correlated electronic and nuclear motion on ultrafast timescales.

However, the real-time dynamics of highly-excited molecules have remained largely unexplored. Conventional synchrotrons could not directly emphasize the 'dynamics' due to the lack of time resolution. Similarly, the multiphoton/strong-field approach with IR/Vis pulses is inadequate, as it preferentially targets the loosely bound electrons and cannot directly access inner electrons.

Fortunately, the table-top XUV pulses obtained through laser high-harmonic generation (HHG) provide an excellent source in terms of photon-energy and time-resolution required to probe fast electron-electron and electron-ion dynamics. In figure 2, we have summarized the essential aspects of laser high-harmonic generation. For a detailed discussion of this process reader is referred to an explanatory article by Kapteyn et al. [23]. Below, we focus on the unique opportunities opened up by the application of HHG source.

The advantages of table-top, attosecond, XUV source are many fold. Firstly, due to short pulse duration (Fig. 2(d), attosecond-few femtosecond), XUV excitation creates highly localized wavepackets such as those shown in figure 1(b). Temporally and spatially localized wavepackets allow ultrafast time-resolved study of excited-state chemical dynamics, naturally providing sub- $\AA$ spatial sensitivity to the reaction coordinate. Secondly, the high-harmonic based XUV (a)
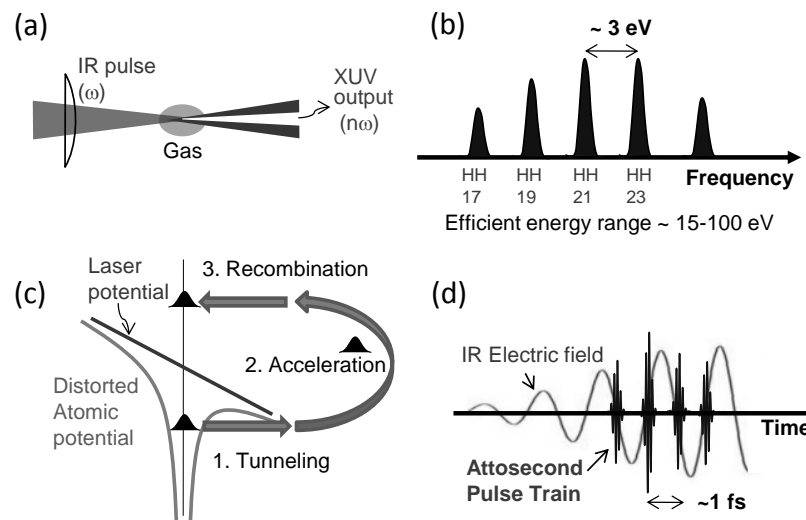

(d)

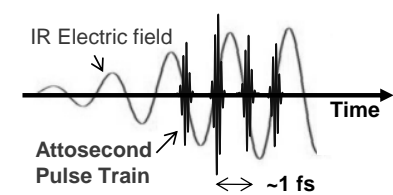

Fig. 2 (a) Intense near-IR femtosecond laser pulse focused on rare gas atoms leads to XUV emission (b) Spectral output is in the form of odd harmonics extending to 100 's eV (c) The generation mechanism is a three step process involving tunneling, acceleration and recollision of electronic wavepacket with core (d) Laser driven recollision mechanism repeats every half-cycle leading to synchronized attosecond duration XUV bursts.

pulses most efficiently generated in the photon energy regime of 10-100 eV (Fig. 2 (b)). This is highly appropriate for probing excited state dynamics as most molecules exhibit peak oscillator strengths in this photon energy regime[24]. Thirdly, the three step mechanism[25-27] (Fig. 2(c)) ensures phase coherence and perfect synchronization between XUV attosecond pulse trains (APTs) (or single attosecond pulses) [28-34] and the driving IR pulses. This intrinsic sub-cycle synchronization allows XUV+IR pump-probe experiments that can time-resolve what happens in the attoseconds or first few femtoseconds of photon-molecule interaction.

As an opening example, we discuss the fragmentation dynamics of $\mathrm{N}_{2}$ molecule, which represents the first measurement of XUV pulse initiated femtosecond molecular dynamics[20]. In this experiment, XUV pulse is used to
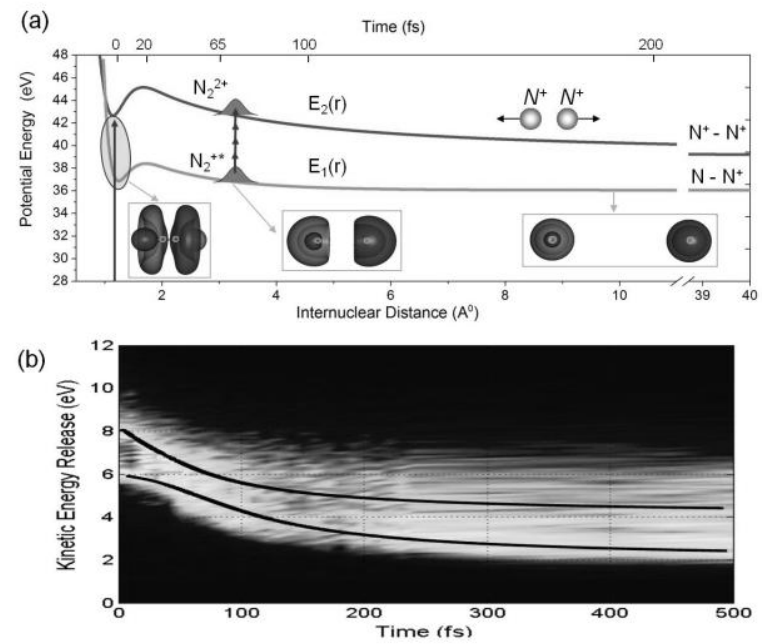

Fig. 3 (a) XUV photon forms excited state $\left(\mathrm{N}_{2}{ }^{+*}\right)$ though electronic shakeup. Time delayed IR probe pulse converts it to dication state $\mathrm{N}_{2}{ }^{2+}$. (b) The kinetic energy release of coincident $\mathrm{N}^{+} / \mathrm{N}^{+}$channel as a function of XUV+IR delay (black lines are fit from theory). The KER variation elucidates the dynamics of loosely bound electron formed in the $4 \sigma_{u}$ orbital during shakeup process. The wave function corresponding to $4 \sigma_{\mathrm{u}}$ electron is shown at three different time delays i.e., internuclear distances. 
create a localized ionic wavepacket $\sim 40 \mathrm{eV}$ above ground state (see Fig. 1 and Fig 3(a)). Using photo-fragment imaging technique discussed in section IV, we observed two main product channels. The inner-valence $\left(2 \sigma_{\mathrm{g}}^{2} 2 \sigma_{\mathrm{u}}^{2}\right)$ ionized molecules fragment into $\mathrm{N}\left(2 \mathrm{~s}^{2} 2 \mathrm{p}^{3}\right)$ and $\mathrm{N}^{+}\left(2 \mathrm{~s}^{2} 2 \mathrm{p}^{2}\right)$. More importantly, certain excited molecular ions $\left(\mathrm{N}_{2}{ }^{+*}\right)$ evolve into ground state $\mathrm{N}^{+}$and an excited neutral atom that has loosely bound $(n=3)$ electron attached to it. The second excitation and fragmentation path is shown as lower potential energy curve in figure 3(a) and it represents an interesting and unexplored relaxation channel.

Using a time-delayed IR pulse, we remove the weaklybound shakeup electron, leading to $\mathrm{N}^{+}-\mathrm{N}^{+}$product channel shown in figure 3(a). This channel was isolated by selecting coincident events pairs with zero center-of-mass momentum. The kinetic energy release (KER) of these events is shown in Fig. 3(b) as a function of time delay. The excellent agreement between theory calculation (black lines) and experimental KER identifies the electronic excited state $\mathrm{N}_{2}{ }^{+*}$ in Figure 3(a) as $4 \sigma_{\mathrm{u}}$ electron-shakeup accompanying the $3 \sigma_{\mathrm{g}}$ ionization.

The sharp decrease in KER over the first $150 \mathrm{fs}$, represents a rapid change from an almost spherically symmetric initial binding potential to a final two-center potential for $4 \sigma_{u}$ electron. The wave function corresponding to anti-bonding $4 \sigma_{\mathrm{u}}$ orbital is shown in Fig. 3(a) below the potential energy

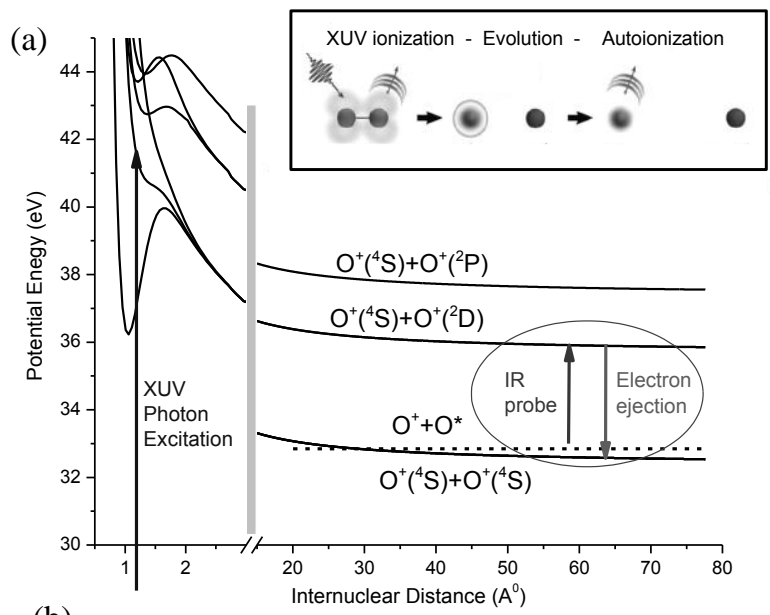

(b)
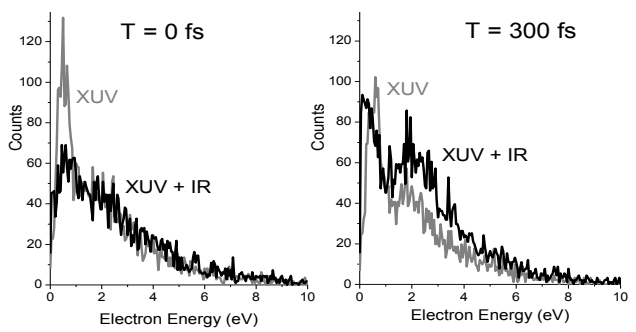

Fig. $4 \mathrm{XUV}$ excitation of $\mathrm{O}_{2}$ molecule. Dashed curve represents the dissociation limit of auto-ionizing $\mathrm{O}_{2}{ }^{+*}$. Solid curves are di-cation dissociation limits. Auto-ionization is allowed for internuclear distances greater than $30 \AA$ corresponding to $\sim 300$ fs delay. Auto-ionization dynamics are time-resolved using IR probe. (b) Electron spectra as a function of time delay show the appearance of $2 \mathrm{eV}$ peak corresponding to onset of autoionization at $300 \mathrm{fs}$. curves. At $\mathrm{r}=1.1 \AA$ there is almost no electron density between the two $\mathrm{N}^{+}$cores. However, at $\mathrm{r}>3 \AA$ the electron density between two cores is substantial, and the two-center nature of the potential is apparent.

In another XUV+IR experiment, Sandhu et al. [19] performed a time resolved study of auto-ionization dynamics of highly-excited $\mathrm{O}_{2}$ molecules. Auto-ionization after XUV photoionization of $\mathrm{O}_{2}$ is a complex multistep process. By interrupting this process with a short IR laser pulse, it was demonstrated that autoionization cannot occur until the internuclear separation of the fragments is greater than $\sim 30$ A. Figure 4 (top-left) shows the XUV excitation, molecular evolution and IR probing steps relevant to this photonmolecule reaction.

Potential energy curves in Figure 4(a) shows FrankCondon region near $1 \AA$ and the dissociation limits corresponding to various internal states of fragments. Energetically favored and symmetry allowed auto-ionization happens through electronic transition between dashed line $\left(\mathrm{O}^{+} / \mathrm{O}^{*}\right)$ and lowest solid curve representing ground state $\left(\mathrm{O}^{+} / \mathrm{O}^{+}\right)$ion fragments, releasing $\sim 0.5 \mathrm{eV}$ electron[35]. As shown in Fig. 4(a), the time-delayed $1.5 \mathrm{eV}$ IR probe photon can be used to interrupt this process by forcing transition to excited ionic state $\left(\mathrm{O}^{+}\left({ }^{4} \mathrm{~S}\right)+\mathrm{O}^{+}\left({ }^{2} \mathrm{D}\right)\right)$. This is followed by distinct electron ejection at energy equals IR photon plus the auto-ionization energy i.e. $1.5 \mathrm{eV}+0.5 \mathrm{eV} \sim 2 \mathrm{eV}$. The appearance of this $2 \mathrm{eV}$ peak at $\sim 300 \mathrm{fs}$ in figure $4(\mathrm{~b})$ confirms the onset of auto-ionization.

I should be mentioned that Feifel et al [35] had performed a coincident electron study in $\mathrm{O}_{2}$, however, these synchrotron experiments did not have time-resolution to uncover the dynamics. Our use of pump-probe XUV+IR spectroscopy enabled direct observation of the transformation of electronically bound states of the molecular-ion into Feshbach resonances of the neutral oxygen atom.

\section{ROLE OF IR FIELDS IN XUV SPECTROSCOPY}

The above examples clearly show that IR pulse plays an important role in time resolving the molecular dynamics of XUV excited states. Obviously, the ultrashort duration (10's femtosecond) of IR pulse and its synchronization with XUV make it an ideal probe pulse. However, the role of IR pulse is more subtle that that. Actually, it is the high field strength associated with IR pulses that ensures the success of ultrafast XUV spectroscopy.

Despite the high oscillator strengths at XUV wavelengths, typical reaction channels associated with highly-excited states exhibit a low cross section. For example, if we ignore the valence ionization, the net photoionization cross-section[36] for $\mathrm{N}_{2}$ at $50 \mathrm{eV}$ is $<3 \mathrm{Mb}$. Hence, for typical parameters such as $1 \mathrm{~mm}$ supersonic gas-jet with density $10^{11}$ molecules/cc, one needs $10^{7}$ XUV photons for a single non-valence event.

Unfortunately, the efficiency of high-harmonic processes is fairly low $\left(<10^{-6}\right)$. Hence, the number of XUV photons 
reaching the interaction region is typically of the order $\sim 10^{7}$ per pulse. This implies that for the $\mathrm{N}_{2}$ case discussed above, each XUV pulse will only prepare $\sim 1$ interesting non-valence excited molecule in the focal volume. To ensure that this excited molecule is indeed probed, the probe needs to have a high flux.

To get a rough idea of probe pulse requirements, let's assume probe photo-absorption cross section $\sim$ few $\mathrm{Mb}$ and focal volume of typical dimensions $100 \mu \mathrm{m} \times 100 \mu \mathrm{m} \times 1 \mathrm{~mm}$. This suggests that in the visible/near-IR range $>10^{13}$ photons per pulse are required. In other words, peak intensity in the range of $10^{12}-10^{13} \mathrm{Wcm}^{-2}$ is required of the probe.

Table I summarizes the estimated non-valence photoexcitation probability in the focal volume for typical molecular beam geometries. The photo-excitation events per laser shot are determined by the number of photons/shot, target number density, interaction length, and the cross section, as follows: $N_{\text {excited }}=N_{\text {photons }} \times\left(n_{\text {molecules }} \cdot L \cdot \sigma\right)$

TABLE I

XUV EXCITATION PROBABILITY

\begin{tabular}{lccc}
\hline \hline Target type & $\begin{array}{l}\text { Target density } \\
\left(\mathrm{cm}^{-3}\right)\end{array}$ & $\begin{array}{l}\text { Cross-section } \\
(\mathrm{Mb})\end{array}$ & $\begin{array}{l}\text { Excitation } \\
\text { events in focal } \\
\text { volume }\end{array}$ \\
\hline $\begin{array}{l}\text { Supersonic } \\
\text { molecular beam }\end{array}$ & $\sim 10^{11}-10^{12}$ & $\sim 1$ & $\sim 0.1-1$ \\
$\begin{array}{l}\text { Supersonic } \\
\text { cluster beam }\end{array}$ & $\sim 10^{10}-10^{11}$ & $\sim 10$ & $<0.1-1$ \\
$\begin{array}{l}\text { Effusive } \\
\text { molecular jet }\end{array}$ & $\sim 10^{12}-10^{14}$ & $\sim 1$ & $\sim 1-100$ \\
\hline $\begin{array}{l}\text { These estimates assume an interaction length } \mathrm{L}=1 \mathrm{~mm}, \text { and } \sim 10^{7} \mathrm{XUV} \\
\text { photons per pulse in the spectral window from } 25-75 \mathrm{eV} .\end{array}$ &
\end{tabular}

Above discussion makes it clear that to get reasonable statistics, a moderate-to-high strength probe field is crucial for XUV spectroscopy. Hence, it is no surprise that most table-top XUV experiments utilize intense probe IR pulses, enabling what we call the XUV+IR spectroscopy.

If the high-field IR pulses are required in XUV spectroscopy, this raises an interesting question, namely, what is the effect of strong transient electric fields on electronic structure of atoms and molecules? As we show in section VI, the XUV+IR spectroscopy in fact provides us an ideal tool to address this question.

\section{EXPERIMENTAL METHODS}

Typical beam line geometry used in XUV+IR experiments involves splitting the intense IR pulse into two parts. One part is used to generate high-harmonic XUV emission in the form of attosecond pulses or pulse trains (Beam 1). The other part of the IR pulse is time-delayed (Beam 2) and is overlapped with XUV in the interaction region. A mirror with a hole recombines the two beams collinearly.

The typical XUV+IR experimental setup is shown in the figure 5(a). High harmonics are generated in a gas filled hollow waveguide (150 $\mu \mathrm{m}$ bore). Waveguide geometry offers

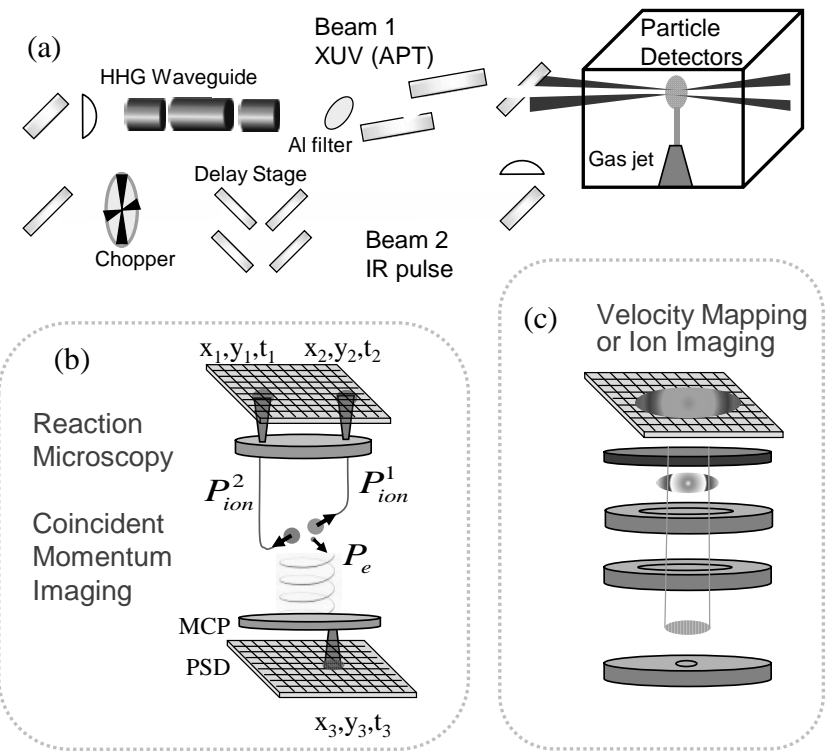

Fig. 5 (a) Ti:Sa laser amplifier produces $30 \mathrm{fs}$ IR pulses at $1 \mathrm{kHz}$ repetition rate with pulse energy $\sim 2 \mathrm{~mJ}$. XUV attosecond pulses train (APT) is obtained by high-harmonic generation in a gas-filled waveguide. XUV and time-delayed IR pulses are focused on to gas target. (b) In case of femtosecond molecular dynamics, photofragments are analyzed in a momentum-imaging reaction microscope consisting of position sensitive delay line detectors (PSD). Particle hits $(\mathrm{x}, \mathrm{y}, \mathrm{t})$ give the 3-D momentum vector of each particle. (c) In attosecond strong field ionization dynamics, we use velocity mapping or spatial ion imaging.

control over spectral and temporal properties of $\mathrm{HHG}[32,37]$ and high conversion efficiency through efficient phase matching [38]. XUV emission is focused on to atomic or molecular targets using multilayer coated or grazing incidence dielectric mirrors[39].

Two types of photo-fragment detection schemes were utilized here. Figure 5(b) shows reaction microscope designed for probing femtosecond molecular dynamics[40]. Velocity map imaging[41] set up in figure 5(c) is optimized for attosecond resolved studies of atomic ionization dynamics.

In the molecular experiments, due to simultaneous population of many excitation channels by the XUV, and the complexity of excited-state potential energy surfaces (PES), the evolution of a specific reaction channel is extremely hard to de-convolve from multitude of fragment that are typically observed in such experiments. In order to implement signal extraction and analysis in molecular experiments, a coincident photo-fragment imaging reaction microscope was developed [39]. In this technique, photo-fragments generated in each laser shot are collected using electric and magnetic fields (Fig. 5(b)). Using position and time sensitive detectors, three dimensional momenta of fragments is then computed on shot to shot basis. Using electron-ion momentum correlations, it was possible to identify and study specific reaction dynamics[39].

To implement attosecond resolved measurements of atomic ionization dynamics with high count rates resulting from effusive gas jet, we collected the electrons/ions produced in the focal region using electrostatic fields and imaged to a 


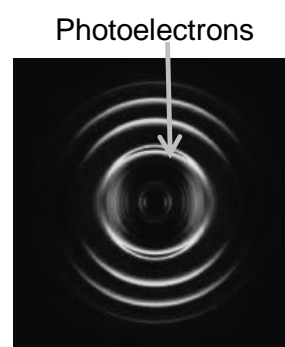

XUV only

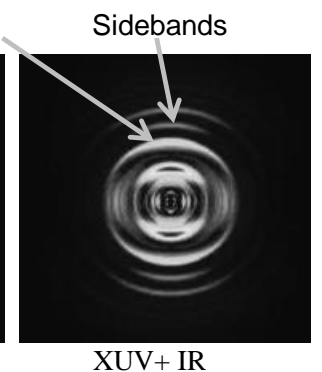

XUV+ IR
Fig. 6 Velocity map imaging of photoelectrons resulting from ionization of Helium atom using XUV pulses generated in Ar filled waveguide. Two cases are shown, XUV alone and XUV+IR. Presence of IR leads to formation of sidebands through dressing of electrons.

MCP-phosphor assembly backed by a CCD camera (Fig. 5(c)). Two different imaging modes are used: 1) velocity map imaging for electrons and 2) one-to-one spatial mapping of ions, from the point of origin in the focal volume and the point of their impact on the detector. The velocity map imaging (VMI) allows high energy resolution, as all fragments with same initial velocity vector are imaged to the same point on the detector regardless of their point of origin in the focus[41]. The spatial imaging mode essentially maps the focal volume on to MCP detector, thus allowing us to discriminate the low energy ions produced under different IR phase and intensity conditions.

XUV spectrum was calibrated using the VMI of photoelectrons produced from Helium. Figure 6 shows the photoelectron rings corresponding to the comb of odd harmonics $\left(2 \omega\right.$ spaced). The first ring corresponds to $17^{\text {th }}$ harmonic which is the first one above the ionization threshold of Helium. In presence of the IR pulse, the continuum energy of photoelectron is modified through IR field induced dressing. This leads to additional rings in the form of sidebands that are spaced by energy of IR photon[28].

\section{INTERACTION REGION: PHASE AND INTENSITy EFFECTS}

In the $\mathrm{XUV}+\mathrm{IR}$ experiments, attosecond resolution is obtained by controlling the relative phase between two fields (figure 7(a)). Thus, for accurate data acquisition, it is of crucial importance that the relative XUV/IR phase remains constant over the interaction region.

As the XUV interaction being a weak field effect, it results in excitation of atoms over the entire path length through the gas jet. In contrast, the strong fields are relevant only in highintensity region of the focal volume, which can be characterized by the Rayleigh length. Hence, one needs to ensure that phase delay between two fields is constant over smaller of the Rayleigh length or the gas jet extent.

It is a well-known fact that an electromagnetic wave going through the focus, experiences a phase change, called the Gouy phase shift [42]. The Gouy phase shift for Gaussian beams is $\pi$, but it can have arbitrary values for more complex profiles [43, 44]. As a result, there is a spatial phase slip (a)

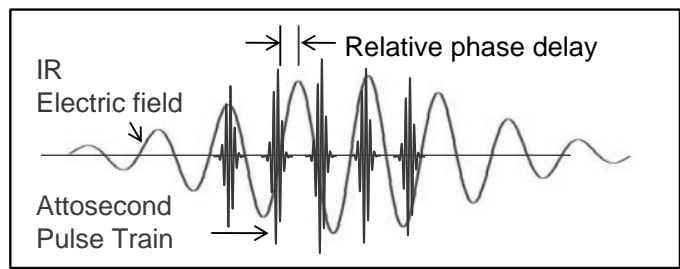

(b)

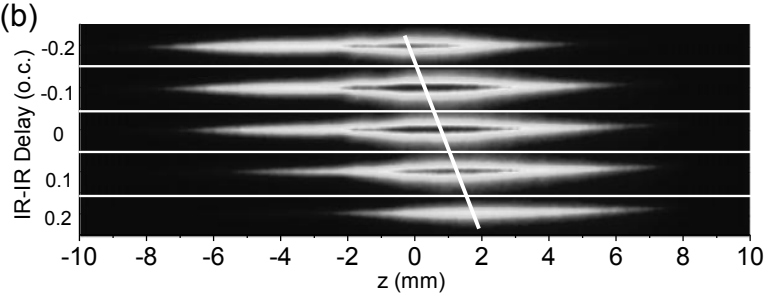

(c)

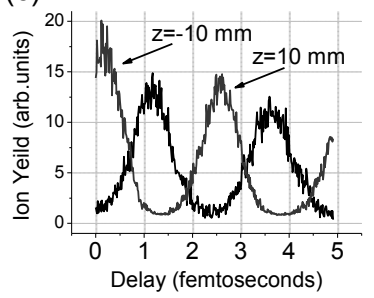

(d)

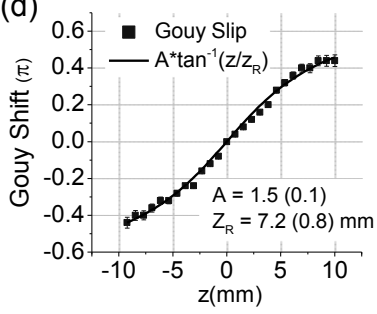

Fig. 7 (a) The relative phase between XUV and IR fields is used a control parameter in attosecond experiments. (b) Two-dimensional ion-images of focal region for different time (phase) delays between the two IR pulses. The peak of the ion signal moves in the z-direction (propagation direction) when the delay is changed, indicating a phase slip along the focus. (c) Oscillations of Xe ion counts as a function of IR/IR delay for two different sections at $\mathrm{z}=$ $10 \mathrm{~mm}$ and $\mathrm{z}=10 \mathrm{~mm}$ are almost $\pi$ out of phase. (d) Calibration of Gouy phase shift as a function of distance along the laser focus.

between XUV and IR fields in the interaction region. This obviously leads to signal averaging over a range of relative phases, adversely affecting the measurement accuracy in XUV+IR experiments. To ameliorate the effects of Gouy phase slip, researchers typically use methods that involve placing the target before/after the focus or limiting of the interaction to a fraction of the Rayleigh range[11, 28].

With increasing attention towards real-time measurement and control of electron dynamics, it becomes important to understand the role of Gouy phase slip in attosecond measurements. Here, we discuss a method[45, 46] for accurate characterization of the phase slip in a typical twobeam focusing geometry shown in figure 5 .

In the XUV+IR experiments (Fig. 5), the Gouy phase shift of XUV (beam 1) is unimportant as the wavelength of XUV is very short and is Rayleigh parameter is very large in comparison to IR field. As a result, the relative phase-slip is purely due to the Gouy shift of the IR pulse (beam 2).

We show that the phase slip of IR pulse can be characterized through spatial imaging of Xe ionization [46]. The aluminum filter in the beam 1 is opened to allow IR output from waveguide to propagate and XUV output is blocked by using a thin glass plate. Of the two IR pulses (beam 1 and beam 2), the weakly focused beam 1 serves as a reference to measure phase slip of beam 2 as a function of longitudinal distance along the propagation direction (i.e. zaxis). The intensities of each of the IR pulses were chosen 
such that ionization happens only in the simultaneous presence of the two fields and the ionization yield at a point in the focal region is depends on the local electric field resulting from two pulses.

For a fixed time delay, as the two overlapping IR pulses propagate through the focus, any change in relative phase between due to the Gouy-phase slip of beam 2 results in the spatial variation of the ion yield. In particular, peak ion yield will occur wherever the two IR fields are 'in-phase'.

Figure 7 (a) shows the spatially resolved ion images obtained for five different time (phase) delays between the two IR pulses. Ion images exhibit a peak, which shifts spatially (along z-direction) as the relative time delay is changed. This is a clear indication of the phase slip between the two IR pulses as they go through the focus $[45,46]$.

Fig. 7(b) shows ion yield vs. time delay for two vertical sections of the ion image corresponding to $\mathrm{z}=-10 \mathrm{~mm}$ and $\mathrm{z}$ $=+10 \mathrm{~mm}$, i.e. before and after the focus. As expected, the ion counts from these two end sections show clear oscillations with a period equal to the laser cycle period of 2.6fs. More importantly, due to the relative phase slip, the oscillations from each end of the interaction region are almost $\pi$ out of phase! Repeating this measurement for many spatial sections along the propagation direction, one can build a map of Gouy phase slip in the focal region, which is shown in Fig. 7(c).

In the absence of knowledge about the exact profile of 'annular' beam 2, the measured phase shift can be fit to an expression, $\phi=A \cdot \tan ^{-1}\left(z / z_{R}\right)$, where multiplier ' $A$ ' characterizes the net effect due Gouy shift of high-order modes[44] constituting the annular beam, and $z_{R}$ is the Rayleigh range. A good fit is obtained for $\mathrm{A}=1.5$ and $z_{R}=$ $7 \mathrm{~mm}$, which is in agreement with the expected Rayleigh range of beam 2. In summary, Fig. 7(c) represents in-situ calibration of the unknown Gouy phase slip of a complex annular beam 2 as a function of distance along the focus, providing us with a phase-meter for precision attosecond experiments. The advantage of this imaging approach is that it allows us to implement spatial selectivity to enhance the contrast of phase dependent XUV+IR pump-probe data, allowing us to uncover previously unobservable phase and intensity effects in XUV+IR ionization.

\section{ATOMS IN STRONG FIELDS}

An important application of attosecond XUV+IR spectroscopy is in the study the atomic ionization dynamics. Motivation behind this line of investigation is to understand and control a fundamental process, such as photo-absorption, on ultrafast timescale. It is known that strong IR fields can substantially alter the atomic structure and can even induce transparency for x-rays [47].

Here, we focus on the control of two-color XUV+IR ionization dynamics on few-femtosecond timescale in a simple system such as Helium atom. The schematic of this

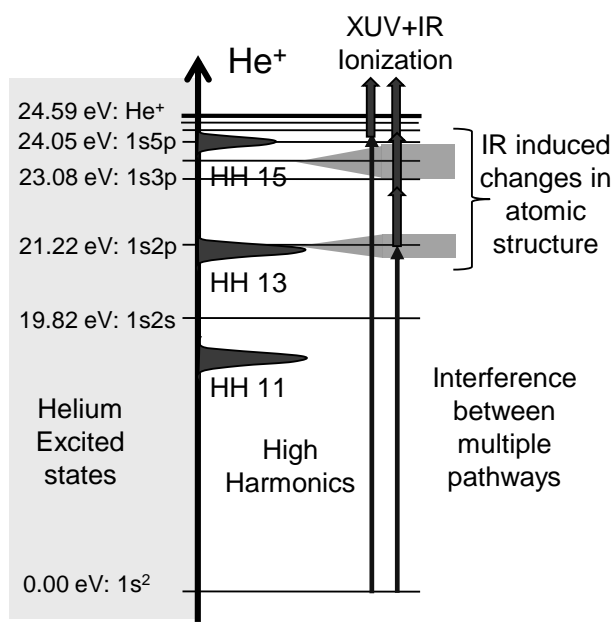

Fig. 8 Excited states of He relative to the high harmonics 11th, 13th and 15th. The two-photon XUV+IR ionization leads to formation of $\mathrm{He}^{+}$ions. Photoionization is affected by IR field induced modification of resonances and dynamic interferences between different ionization pathways.

interaction is shown in figure 8 . Helium has fairly discrete excited state spectrum. To access the He excited states, we use the attosecond pulse train (APT) corresponding to $11^{\text {th }}, 13^{\text {th }}$, $15^{\text {th }}$ harmonics obtained from Xe filled waveguide. These harmonics lie below the ionization threshold of Helium (24.6 $\mathrm{eV}$ ), and depending upon the energy overlap between harmonics and excited states, the APT primarily populates the resonant 'np' excited states. Above threshold $17^{\text {th }}$ harmonic has tiny presence in the XUV spectrum; implying negligible direct ionization by APT. The presence of a strong femtosecond IR pulse dynamically modifies the electronic states and tuning the phase and intensity of IR field should provide a certain level of control over ionization pathways.

In a recent experiment[48], it was observed that the exact harmonic spectrum and the strength of IR field plays an important role in the efficiency of the ionization process. In a separate experiment[14], it was pointed out that the IR ionization of electron wavepackets excited by consecutive XUV pulses in APT can lead to interference in the continuum, modulating the efficiency of this process as a function of relative phase between IR and APT.

Detailed analysis of transient ionization dynamics can be undertaken by the full solution of a time-dependent Schrodinger's equation. However, relative long IR pulse duration ( $40 \mathrm{fs})$ and periodic nature of interaction provides a natural theoretical framework to model this problem. This formulation [49] is based on the formation of Floquet states in a strong periodic IR field.

The dynamics of an atom in a periodic external field are governed by the following time dependent Schrodinger equation (in atomic units):

$$
i \frac{\partial \psi(t)}{\partial t}=\left[H_{0}+V(t)\right] \psi(t)
$$

$H_{0}$ is the field free atomic potential and $V(t)$ is periodic potential. In Floquet formalism[49], the solution can be written in the form 


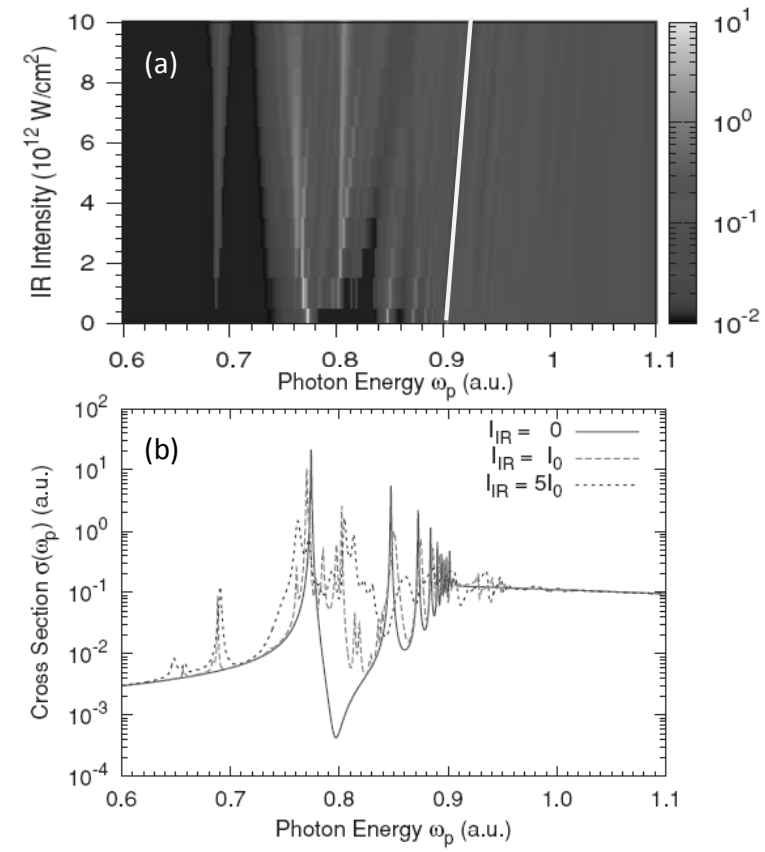

Fig. 9 (a) IR modified photo-absorption cross sections in Helium as a function of the IR intensity. The white line starting at $\omega_{\mathrm{p}}=0.9$ a.u. shows the variation of ionization threshold due to the ponderomotive shift. (b) Lineouts of photoabsorption cross section at three intensities in terms of $\mathrm{I}_{0}=10^{12} \mathrm{Wcm}^{-2}$.

$$
\psi_{\alpha}(t)=e^{-i \varepsilon_{\alpha} t} \sum_{n=-\infty}^{n=+\infty} e^{-i n \omega t} \phi_{\alpha, n}
$$

where $\varepsilon_{\alpha}$ is the quasi-energy, $\phi_{\alpha, n}$ is the time independent wavefunction for the $\mathrm{n}^{\text {th }}$ Fourier component of the $\alpha$ Floquet state and $\omega$ is the frequency of the external periodic field. Thus, in presence of a laser field, electronic structure can be conveniently described by Floquet states, where each bound state is associated with many one-photon spaced sidebands.

If the external field is strong enough to modify the excited and continuum states, but weak enough not to perturb the ground state, we can write the photo-absorption cross section for transition from ground state to a given Floquet state as

$$
\sigma\left(\omega_{p}\right)=\frac{4 \pi \omega_{p}}{c} \operatorname{Im} \sum_{\alpha, n} \frac{\left|\left\langle\psi_{g}|d| \phi_{\alpha, n}\right\rangle\right|}{\left(\varepsilon_{\alpha}+n \omega\right)-\left(\varepsilon_{g}+\omega_{p}\right)-i \eta}
$$

Here $\omega_{\mathrm{p}}$ is the frequency of $\mathrm{XUV}, d$ is the dipole operator, $\Psi_{g}$ is ground state wavefunction, $\varepsilon_{g}$ is the ground state energy and $\eta$ is an infinitesimal parameter used to represent adiabatic switching of the interaction.

In principle, the time dependent wave function $\Psi_{\alpha}(\mathrm{t})$ can be obtained by solving the time-independent Floquet equation, however in practice it is highly computation intensive task. Instead, we use a simpler method developed by Tong et al. [50] to calculate the effects of IR field on electronic structure of Helium atom.

Figure 9 summarizes the behavior of atomic resonances as a function of probing XUV photon energy and peak IR intensity. Clearly, the discrete resonances evolve into a complicated structure in intense laser fields. The low-lying $2 p$ state (at 0.78 a.u.) exhibits a small shift, whereas the higher

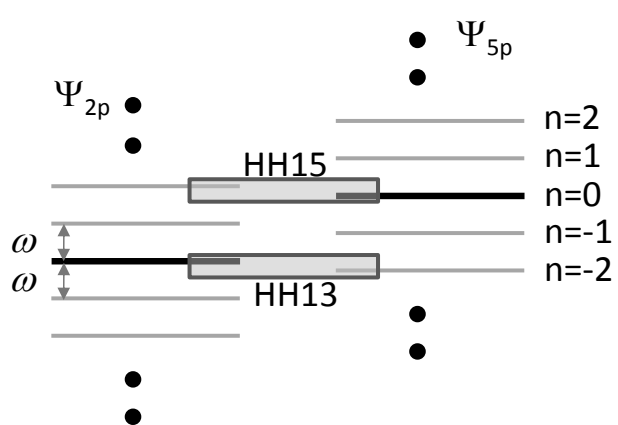

Fig. 10 Energy structure of two Floquet states corresponding to $2 p$ and $5 p$ atomic states under the influence of a strong periodic $(\omega)$ IR field. Relative positions of high harmonic 13 and 15 are also shown in the diagram.

excited-state manifold is completely altered by strong fields and the width of excited states becomes comparable to or even larger than the spacing between unperturbed energy levels. As expected, the near-threshold shifts can be approximated by ponderomotive energy associated with IR field.

Figure 9 clearly shows that the photo-absorption cross section at a given frequency can be modified by many orders of magnitude through application of IR fields. Importantly, this modification can be achieved on ultrafast (femtosecond) timescale! In the following section, we demonstrate the use of attosecond XUV+IR spectroscopic methods to understand and control the photoionization dynamics.

\section{XUV+IR IONIZATION DYNAMICS}

\section{A. Interfering paths}

Figure 8 shows that $13^{\text {th }}$ and $15^{\text {th }}$ harmonic are two most relevant excitation frequencies in Helium. Couple of IR dressed Floquet manifolds that can be excited by these frequencies are shown in figure 10. These Floquet manifolds correspond to $2 p$ and $5 p$ states. Each state has several Floquet components separated by IR laser frequency $(\omega)$.

To begin the analysis of XUV+IR dynamics let us assume a single Floquet state is dominant. The ionization proceeds through coherent channels represented by the $\omega$-spaced Floquet components of that state. These coherent paths interfere, and the resultant ionization probability depends on XUV-IR delay. The time-dependence of the ionization signal can be deduced from the following discussion.

Assuming that both XUV and IR field are linearly polarized along $z$-direction, the external potential in equation (1) is $V(t)=-z\left[E_{I R}(t)+E_{X U V}(t)\right]$. If XUV APT pulse arrives at time delay $\tau$ relative to IR pulse, we can write XUV field as

$$
E_{X U V}(t)=\int f\left(\omega_{X U V}\right) e^{-i \omega(t-\tau)} d \omega_{X U V}
$$

where $f\left(\omega_{X U V}\right)$ represents the energy content of XUV pulse (i.e. high harmonic spectra) and also determines the attosecond time structure. The transition amplitude from the ground state to a Floquet state in IR field can be written as 


$$
M_{g \rightarrow \alpha}=\int_{-\infty}^{+\infty}\left\langle\psi_{\alpha}(t)\left|z E_{X U V}(t)\right| \psi_{g}(t)\right\rangle d t
$$

Substituting for $\Psi_{\alpha}(\mathrm{t})$ from equation (2); and $E_{X U V}(\mathrm{t})$ from equation (4) we are led to transition matrix element

$$
\begin{aligned}
M_{g \rightarrow \alpha}= & \sum_{n}\left\langle\phi_{\alpha, n}|z| \phi_{g}\right\rangle \\
& \times \iint_{-\infty} f\left(\omega_{X U V}\right) e^{i\left(\varepsilon_{\alpha}+n \omega-\omega_{X U V}-\varepsilon_{g}\right) t} e^{-i \omega_{X U V} \tau} d \omega_{X U V} d t
\end{aligned}
$$

Implementing the integration, we get matrix element proportional to IR and XUV dependent terms as

$$
M_{g \rightarrow \alpha} \propto \sum_{n}\left\langle\phi_{\alpha, n}|z| \phi_{g}\right\rangle f\left(\omega_{n}\right) e^{i n \omega \tau}
$$

where $\omega_{X U V}=\omega_{n}=\varepsilon_{\alpha}+n \omega-\varepsilon_{g}$.

Under our experimental conditions, we have two main

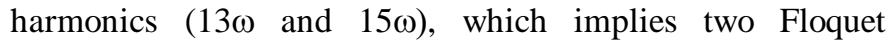
components contribute to ionization. Removing the common phase terms, the probability of ionization can be written as

$$
P(\tau)=\left|M_{13} f_{13}+M_{15} f_{15} e^{-i(2 \omega \tau)}\right|^{2}
$$

The matrix elements between ground state and two Floquet components $\left(\mathrm{M}_{13}\right.$ and $\left.\mathrm{M}_{15}\right)$ depend on the IR intensity. Equation 8 shows that the interference between two Floquet components will modulate the ionization probability twice per IR cycle as a function of time delay i.e. with $2 \omega$ frequency. Now, we compare the understanding gained from this Floquet formalism with our experimental observations.

Using experimental setup described in figure 5, we perform attosecond-resolved imaging of two-color (XUV+IR) ionization of He. This process has been focus of many recent investigations[14, 48, 51-53]. However, here we obtain spatially resolved $\mathrm{He}^{+}$ion-yield in the focal volume as a function XUV-IR delay to illustrate the important from phase averaging. Figure 11 plots the ion-yield for two vertical sections of the ion image, as well as the entire focal region. These positions of $\mathrm{z}=10 \mathrm{~mm}$ and $\mathrm{z}=0 \mathrm{~mm}$ sections can be understood focal volume image in figure 7. In each section, we observe $2 \omega$ or half-cycle oscillations as expected twocomponent Floquet interference.

However, the important thing is that the oscillations in the section after the focus $(\mathrm{z}=10 \mathrm{~mm})$ are almost completely outof-phase with respect to the oscillations in the center section $(\mathrm{z}=0)$. Thus, unlike the IR+IR case (Fig. 7(c)), where the oscillations from two end-sections were out-of-phase, the oscillations in XUV+IR case go out-of-phase over half the spatial distance. The difference stems from the fact that due to $2 \omega$ oscillation of XUV+IR ionization signal, $\pi / 2$ Gouy phase shift of the IR pulse (beam 2) relative to the attosecond XUV bursts (beam1) results in a completely opposite physical situation. Gouy phase shift curve for Beam 2 in figure 7(d) shows that $\mathrm{z}=10 \mathrm{~mm}$ and $\mathrm{z}=0 \mathrm{~mm}$ points differ in phase by $\sim \pi / 2$, which explains the observed behavior.

Furthermore, if we compare the ion yield oscillation in a selected section of the focal region to the oscillation in the

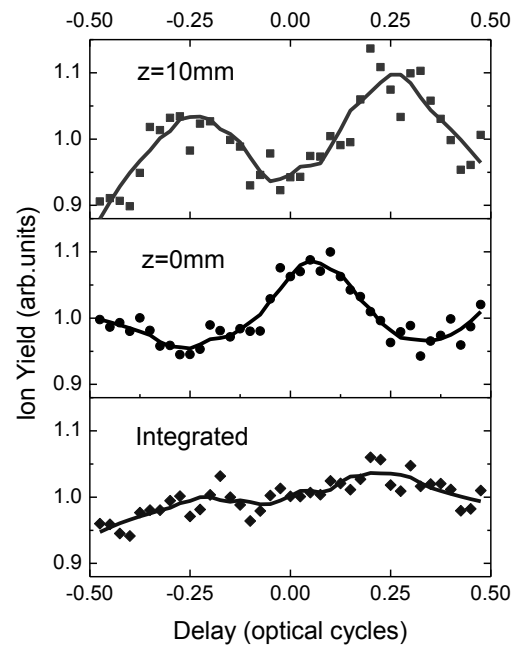

Fig. 11 Helium ion yield as a function of XUV-IR delay for sections $\mathrm{z}=$ $10 \mathrm{~mm}$ and $\mathrm{z}=0 \mathrm{~mm}$ along with spatially integrated yield over the entire focus. Period of oscillations is half-IR-cycle. End section and the center of the focal region are out of phase, leading to loss of contrast in the integrated data.

spatially integrated yield from the entire focal region, we observe that the modulation contrast is much poor in the integrated yield (Fig. 11(c)). The loss of the modulation contrast in integrated data is due to the averaging over different XUV-IR relative phases (delays). This phase averaging in the focus negatively affects most attosecond pump-probe experiments to a certain degree. In fact, in some cases this averaging can mask the interesting phase dependent effects. The imaging technique described in section $\mathrm{V}$ provides a snapshot of phase evolution in the entire focal region and allows us to implement spatial selectivity to enhance signal contrast. Furthermore, the spatial gating of phase-slip allows us to uncover previously unobserved effects.

\section{B. Phases and amplitudes of XUV+IR transitions}

The amplitude and phase of $2 \omega$ oscillations discussed above encodes information on the relative phases and amplitudes of transition matrix elements. We aim to measure the dependence of transition matrix elements on the IR field in a time resolved fashion.

The numerical calculations of XUV+IR ionization in presence of $13^{\text {th }}$ and $15^{\text {th }}$ harmonics along with strong IR field are discussed by Tong et al. in [54]. The calculation results clearly illustrate the dependence of the amplitude and phase of $2 \omega$ ion-yield oscillations on IR intensity. Figure 12(a) shows that the $2 \omega$ oscillation contrast strongly depends on the peak IR intensity. Oscillation amplitude exhibits maximum value for $5 \mathrm{I}_{0}$ and is lower for $\mathrm{I}_{0}$ and $10 \mathrm{I}_{0}$. Importantly, the phase of $\mathrm{I}_{0}$ and $10 \mathrm{I}_{0}$ curve are same, however the $5 \mathrm{I}_{0}$ curve is shifted in phase relative to the other two.

The continuous variation of amplitude and phase with intensity is depicted in figure 12(b). As intensity increases, the oscillation amplitude increases to maximum value at $6 \mathrm{I}_{0}$ and then decreases to minimum value at $10 \mathrm{I}_{0}$. In additional, we notice a phase jump or time shift of 0.25 optical cycles 

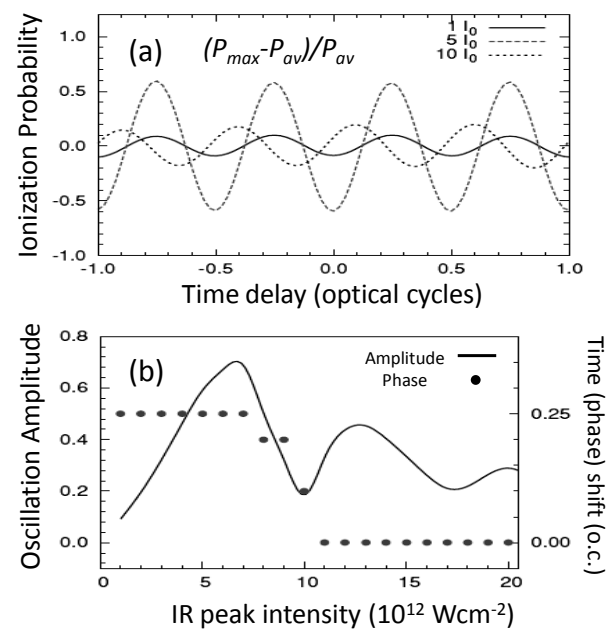

Fig. 12 (a) Normalized ionization probability as a function of XUV-IR time delay for different IR intensities in terms of $\mathrm{I}_{0}=10^{12} \mathrm{Wcm}^{-2} . \mathrm{P}_{\mathrm{av}}$ is the average of ionization probability over one optical cycle. Ionization oscillation amplitude as a intensity (solid line). The time (phase) of oscillations in units of optical cycles is shown (solids dots).

between oscillations at lower and higher intensities. In terms of $2 \omega$ ion-yield oscillations, this shift corresponds to a $\pi$ phase jump.

Figure 12 encodes important Floquet dynamics. For the $13^{\text {th }}$ and $15^{\text {th }}$ harmonic energies used in this calculation, it emerges that $2 p$ and $3 p$ Floquet manifolds both play a role in the ionization dynamics. At lower intensities photoionization is mainly through $3 \mathrm{p}$ Floquet state populated by the $15^{\text {th }}$ harmonic with small contribution from the $13^{\text {th }}$ harmonic. As IR intensity increases, due to ac Stark shift, the transition strength to $3 p$ state by $15^{\text {th }}$ harmonic increases. At the same time, the transition strength to the sideband ( $2 \omega$ Floquet component) of $3 p$ increases even faster, since coupling to Floquet components is IR intensity dependent. Further, the increasing IR intensity, the ac Stark shift moves the $2 p$ Floquet state even farther away from resonance, suppressing its contribution. The factors lead to increase in oscillation amplitude with increasing IR intensity. As we increase the intensity even further, due to Stark shifts, the transitions to both Floquet states become important. However, the two contributions are not in phase, therefore cancellation between the two reduces the oscillation amplitude.

Furthermore, the $\pi$ phase jump on right hand side of figure 12(b) is a manifestation of change in relative phases between transition matrix elements. At low IR intensities, 3p Floquet state is important and the transition matrix elements to this state, i.e. $\mathrm{M}_{13}$ and $\mathrm{M}_{15}$ in equation 8 have a phase difference of $\pi$ (opposite sign). At high IR intensities, $2 \mathrm{p}$ Floquet state is important and for transition to $2 \mathrm{p}$ manifold the transition matrix elements by $13^{\text {th }}$ and $15^{\text {th }}$ harmonic have a phase difference of zero (same sign). Below, we show that IR intensity dependence of magnitudes and relative phases of transition matrix elements is clearly visible in the experiments.

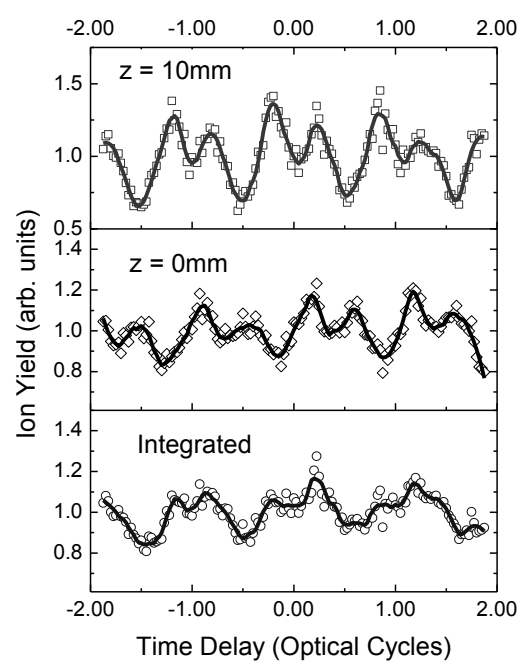

Fig. $13 \mathrm{He}$ ion yield with of XUV and two IR pulses. Oscillations at $2 \omega$ are superposed on the $\omega$ oscillation. The relative phase between the two oscillations leads to the observed time-dependent asymmetric oscillations structure. Spatially discriminated data from the end and center sections shows Gouy phase shift. The oscillations structure is washed out in the integrated data due to Gouy-phase averaging.

\section{C. $X U V+I R+I R$ experiments}

In principle, time-resolved XUV+IR measurements encode the information on intensity dependence of the electronic structure. However, due to experimental constraints such as fluctuations and interferometric instabilities it is very hard to extract the relative phases and amplitudes of $2 \omega$ oscillations in a straightforward manner. In order to extract these quantities in a time resolved manner, we propose the use of XUV plus two-IR pulse technique[46, 48, 55] to convert the phase information to amplitudes of ion-yield oscillations.

In this technique $[46,55]$, we use the interference between two IR pulses to create a reference frequency $\omega$, which allows us to observe the phases and amplitude of $2 \omega$ oscillations in a robust manner. Experimentally, this is achieved by simply opening the aluminum filter to allow HHG driving IR pulse to co-propagate with the high harmonics. Referring to figure 5, probe IR pulse follows the usual beam 2 path, whereas the second IR pulse co-propagates with the XUV bursts in beam 1. Since the XUV APT is generated by the co-propagating IR, both are inherently phase-locked. The co-propagating IR pulse is weaker than the external probe IR, but strong enough to cause modulation of signal at $\omega$.

Another important aspect of our technique is Gouy phase gating, which removes any averaging due to phase slip and hence allows us to extract phases and amplitudes with an unprecedented resolution. Figure 13 shows the ion yield as a function of delay between XUV+IR (Beam 1) and IR (Beam 2). As before, we plot the ion yields corresponding to two vertical sections $(\mathrm{z}=10 \mathrm{~mm}$ and $\mathrm{z}=0 \mathrm{~mm})$ along with the spatially integrated ion yield over the entire focus. We observe that the oscillations occur with a periodicity of one IR cycle and a small dip in the middle of each cycle is clearly 
visible, thus introducing half-cycle or $2 \omega$ periodicity. The spatially discriminated sections show a distinct asymmetry between peaks on either side of this dip. This structure was predicted recently in [48], but was not been experimentally observed before. Clearly, this detailed structure showing halfcycle asymmetry effect is washed out in the spatially integrated data due to Gouy phase averaging. This problem, often understated in experiments, leads to substantial loss of signal contrast in attosecond experiments and can wash out signatures of interesting quantum processes.

Next we measure the intensity dependence of ionization in XUV+2IR experiments. Figure 14 shows that $\omega$ and $2 \omega$ signals as function of time delay for multiple probe intensities. At low intensities or at large negative time delays, the interference between different Fourier components of the Floquet state i.e. $2 \omega$ oscillation is very small, hence the net ionization signal has one-cycle periodicity. As probe intensity increases, the $2 \omega$ interference between Floquet components becomes important. This can also be seen from the fact that oscillations develop half-cycle periodicity earlier at higher intensities.

Importantly, we observe that relative phase between $\omega$ and $2 \omega$ oscillations can changes very fast during the interaction. If we focus on the transition region (rectangular box) on the top panel of figure 15, we see that oscillation structure is completely opposite before and after. Before -8 o.c. the structure shows asymmetric high peak-low peak variation. After -6.0 o.c. the asymmetry becomes low peak-high peak. This clearly represents a $\pi$ phase change of the $2 \omega$ interference signal.

Our results show a phase jump similar to the one predicted in theoretical calculation shown in figure 12. This represents change in relative phase between matrix elements $M_{13}$ and $\mathrm{M}_{15}$ associated with Floquet components. Even more significant is the fact that this change happens in about one optical cycle or less, which is an extremely fast transfer of ionization pathway from one channel to another.

To summarize, XUV+IR ionization presents a rich topic for investigation of the attosecond dynamics of strong field ionization. Our results show that it is possible to extract amplitudes of various interfering pathways and control the ionization dynamics over timescales corresponding to suboptical cycle or few hundred attoseconds.

\section{New DEVELOPMENTS AND APPLICATIONS}

Advances in laser technology have enabled access to ever shorter attosecond pulses. Furthermore, the use of long wavelength drivers allows phase matching at very high photon energies approaching keV[56-58], where even sub-10 attosecond pulses can be produced. Such pulses by the virtue of their high energy and short pulse duration can enable access to even core shell electrons.

Another important direction where progress is happening

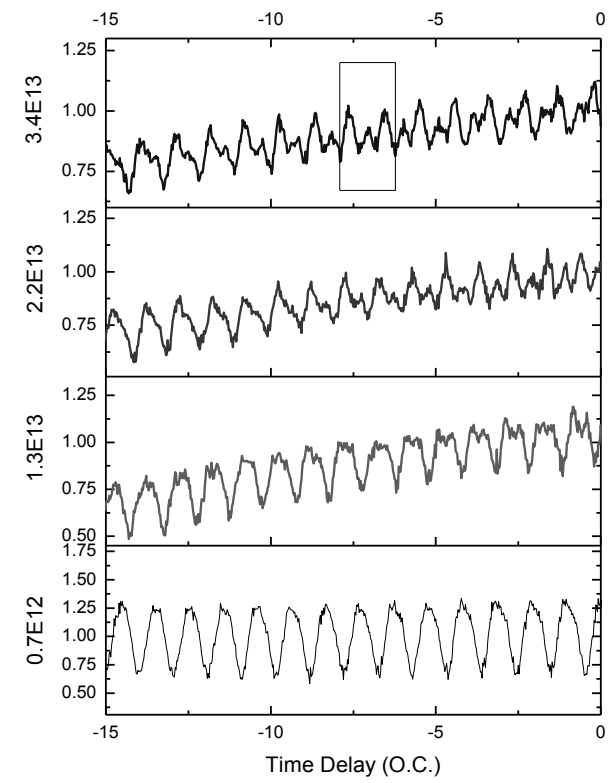

Fig. $14 \mathrm{XUV}+2 \mathrm{IR}$ ionization dynamics in $\mathrm{He}$ as a function of probe IR intensity. Copropagating IR intensity is $\sim 5 \times 10^{12} \mathrm{Wcm}^{-2}$. Notice the oscillation amplitude and phase changes with time delay on few-femtosecond time scale. The box in the top panel $\left(\mathrm{I}_{\text {probe }}=3.4 \times 10^{13} \mathrm{Wcm}^{-2}\right)$ at -7.5 o.c. at time delay shows $\pi$ phase jump similar to the prediction in figure 12 .

is in the improvement of the flux of table-top XUV sources through development of high repetition-rate lasers. Apart from the table-top laser based techniques, upcoming free electron laser (FEL) facilities are also being used for timeresolved experiments. By design FEL sources have high photon densities allowing experiments to probe strong-field response in the $\mathrm{x}$-ray regime.

Assuming a $500 \mathrm{eV}, 10$ attosecond source which is not too far away, we can envision direct observation of electronic excitation and relaxation dynamics. One important example of correlated relaxation dynamics concerns the decay processes where an electron is spontaneously ejected into continuum. These processes include autoionization, Auger decay, Interatomic Coulombic Decay (ICD)[59], etc. These mechanisms are strongly affected by and, in turn, influence the inter-nuclear dynamics. For example, the autoionization rate strongly depends on internuclear distance. Similarly, the Auger process is strongly affected by the changes in valence electron distribution that accompany the molecular evolution in a chemical reaction. The ICD process which occurs efficiently in Van der Waals and hydrogen bonded systems is intimately tied to nuclear coordinates[60]. While these processes are understood theoretically, the real-time experiments are still lacking. The time-of-birth, energy and angular momentum of continuum electrons encode crucial information about the nature of potential and time evolution of molecular wavepacket. In order to tag the 'electron birth', laser pulse streaking can be used to modify the electron momentum at the time of its emergence in continuum due to the momentum impulse provided by the IR probe (Fig. 15). 
Monitoring the electron ejection and obtaining its energy and angular distribution, one can understand electronic relaxation and it dependence on molecular and external potentials e.g. the strong fields. Development along these directions could lead to interesting time domain analogs of high successful molecular characterization methods such as Electron Spectroscopy for Chemical Analysis or ESCA.

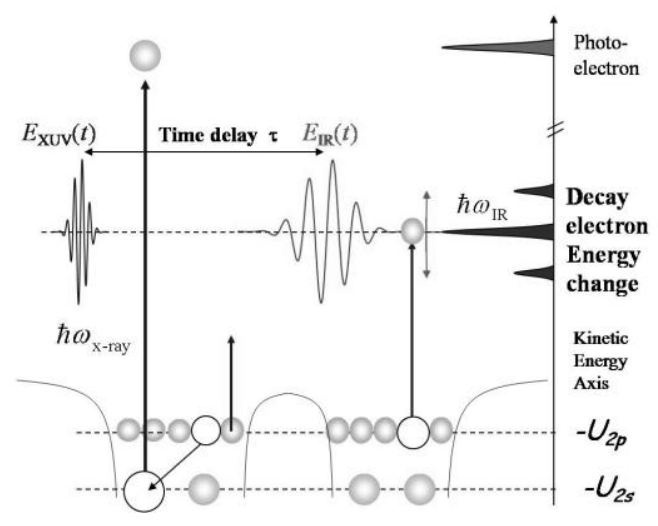

Fig. 15 Schematic for IR streaking of Auger, ICD or autoionization electrons in XUV excited molecules. For example, XUV ionized dimer system exhibits time delayed electron decay. Kinetic energy of photoelectron and decay electron is shown on right. IR streaking field when timed correctly to instant of electron birth leads to observable modification of electron energy/momentum in the form of sidebands.

\section{CONCLUSION}

In summary, the ultrafast XUV+IR spectroscopy combines the strengths of conventional weak-field and strong-field spectroscopies, thus offering a unique tool to understand and control the quantum dynamics in atoms, molecules and materials on the natural timescales of electrons.

Some of the interesting processes that can be investigated through time-resolved XUV spectroscopy are non-adiabatic effects in dissociation, electron-electron correlations in double ionization, electronic relaxation timescales and their dependence on dynamic structure. Such experimental investigations are crucial to understanding of non-equilibrium few-body interactions, and should provide pointers for manipulation of energy flow at the level of chemical dynamics.

The insight into attosecond resolved XUV+IR ionization gives us a handle on the dynamic evolution of electronic structure in the strong field. Our technique allows an accurate characterization of the Gouy phase slip in the focal volume and its effect on the attosecond XUV/IR in atoms. We envision that additional phase dependent effects can be revealed by spatial gating of single-shot ion images, thus turning the deleterious phase slip into a useful tool.

The knowledge gained from these studies, particularly the attosecond control of photo excitation and photoionization dynamics, could serve to open the doors for development of a new generation of ultrafast devices.

\section{ACKNOWLEDGMENT}

We would like to acknowledge Henry Kapteyn, Margaret Murnane, Etienne Gagnon, Lew Cocke, Robin Santra, Predrag Ranitovic, Phay Ho, N. Toshima and Niranjan Shivaram for their substantial contributions to this work.

\section{REFERENCES}

[1]
F. Krausz and M. Ivanov, "Attosecond physics," Reviews of Modern Physics, vol. 81, pp. 163-234, 2009.

M. Uiberacker, et al., "Attosecond real-time observation of electron tunnelling in atoms," Nature, vol. 446, pp. 627-632, 2007. M. F. Kling and M. J. J. Vrakking, "Attosecond electron dynamics," Annual Review of Physical Chemistry, vol. 59, pp. 463-492, 2008. T. Remetter, et al., "Attosecond electron wave packet interferometry," Nature Physics, vol. 2, pp. 323-326, 2006.

P. H. Bucksbaum, "The future of attosecond Spectroscopy," Science, vol. 317, pp. 766-769, 2007.

H. Kapteyn, O. Cohen, I. Christov, and M. Murnane, "Harnessing attosecond science in the quest for coherent X-rays," Science, vol. 317, pp. 775-778, 2007.

T. Brabec and F. Krausz, "Intense few-cycle laser fields: Frontiers of nonlinear optics," Reviews of Modern Physics, vol. 72, pp. 545-591, 2000.

A. Lhuillier and P. Balcou, "High-Order Harmonic-Generation in Rare-Gases with a 1-Ps 1053-Nm Laser," Physical Review Letters, vol. 70, pp. 774-777, 1993.

Z. H. Chang, A. Rundquist, H. W. Wang, M. M. Murnane, and H. C. Kapteyn, "Generation of coherent soft X rays at $2.7 \mathrm{~nm}$ using high harmonics," Physical Review Letters, vol. 79, pp. 2967-2970, 1997. A. Scrinzi, M. Y. Ivanov, R. Kienberger, and D. M. Villeneuve, "Attosecond physics," Journal of Physics B-Atomic Molecular and Optical Physics, vol. 39, pp. R1-R37, 2006.

P. Agostini and L. F. DiMauro, "The physics of attosecond light pulses," Reports on Progress in Physics, vol. 67, pp. 1563-1563, 2004.

P. B. Corkum and F. Krausz, "Attosecond science," Nature Physics, vol. 3, pp. 381-387, 2007.

M. Drescher, et al., "Time-resolved atomic inner-shell spectroscopy," Nature, vol. 419, pp. 803-807, 2002.

P. Johnsson, J. Mauritsson, T. Remetter, A. L'Huillier, and K. J. Schafer, "Attosecond control of ionization by wave-packet interference," Physical Review Letters, vol. 99, pp. 233001, 2007.

J. Mauritsson, et al., "Coherent electron scattering captured by an attosecond quantum stroboscope," Physical Review Letters, vol. 100, pp. -, 2008.

L. Nugent-Glandorf, et al., "Ultrafast time-resolved soft x-ray photoelectron spectroscopy of dissociating Br-2," Physical Review Letters, vol. 8719, pp. 193002, 2001.

T. Pfeifer, et al., "Time-resolved spectroscopy of attosecond quantum

dynamics," Chemical Physics Letters, vol. 463, pp. 11-24, 2008.
L. Miaja-Avila, et al., "Laser-assisted photoelectric effect from surfaces," Physical Review Letters, vol. 97, pp. 113604, 2006.

A. S. Sandhu, et al., "Observing the Creation of Electronic Feshbach Resonances in Soft X-ray-Induced O-2 Dissociation," Science, vol. 322, pp. 1081-1085, 2008.

E. Gagnon, et al., "Soft X-ray-driven femtosecond molecular dynamics," Science, vol. 317, pp. 1374-1378, 2007.

A. H. Zewail, "Femtochemistry: Atomic-scale dynamics of the chemical bond," Journal of Physical Chemistry A, vol. 104, pp. 5660-5694, 2000.

A. Stolow, A. E. Bragg, and D. M. Neumark, "Femtosecond timeresolved photoelectron spectroscopy," Chemical Reviews, vol. 104, pp. 1719-1757, 2004.

H. C. Kapteyn, M. M. Murnane, and I. P. Christov, Physics Today, vol. 58, pp. 39, 2005. 
[24] Y. Hatano, "Interaction of vacuum ultraviolet photons with molecules. Formation and dissociation dynamics of molecular superexcited states," Physics Reports-Review Section of Physics Letters, vol. 313, pp. 110-169, 1999.

[25] P. B. Corkum, "Plasma Perspective on Strong-Field Multiphoton Ionization," Physical Review Letters, vol. 71, pp. 1994-1997, 1993.

[26] M. Lewenstein, P. Balcou, M. Y. Ivanov, A. Lhuillier, and P. B. Corkum, "Theory of High-Harmonic Generation by Low-Frequency Laser Fields," Physical Review A, vol. 49, pp. 2117-2132, 1994.

[27] J. L. Krause, K. J. Schafer, and K. C. Kulander, "High-Order Harmonic-Generation from Atoms and Ions in the High-Intensity Regime," Physical Review Letters, vol. 68, pp. 3535-3538, 1992.

[28] P. M. Paul, et al., "Observation of a train of attosecond pulses from high harmonic generation," Science, vol. 292, pp. 1689-1692, 2001.

[29] R. Kienberger, et al., "Sub-femtosecond X-ray pulse generation and measurement," Applied Physics B-Lasers and Optics, vol. 74, pp. S3-S9, 2002.

[30] A. Baltuska, et al., "Attosecond control of electronic processes by intense light fields," Nature, vol. 421, pp. 611-615, 2003.

[31] I. P. Christov, R. Bartels, H. C. Kapteyn, and M. M. Murnane, "Attosecond time-scale intra-atomic phase matching of high harmonic generation," Physical Review Letters, vol. 86, pp. 5458$5461,2001$.

[32] A. S. Sandhu, et al., "Generation of sub-optical-cycle, carrierenvelope-phase - insensitive, extreme-uv pulses via nonlinear stabilization in a waveguide," Physical Review A, vol. 74, pp. 061803(R), 2006.

[33] G. Sansone, et al., "Shaping of attosecond pulses by phase-stabilized polarization gating," Physical Review A, vol. 80, pp. 063837, 2009.

[34] H. Mashiko, et al., "Extreme ultraviolet supercontinua supporting pulse durations of less than one atomic unit of time," Optics Letters, vol. 34, pp. 3337-3339, 2009.

[35] R. Feifel, J. H. D. Eland, and D. Edvardsson, "Valence double ionization of O-2 at photon energies below and above the molecular double ionization threshold," Journal of Chemical Physics, vol. 122, pp. 144308, 2005.

[36] S. Krummacher, V. Schmidt, and F. Wuilleumier, "Inner-Shell Photoionization in Molecules - the Nitrogen Case," Journal of Physics B-Atomic Molecular and Optical Physics, vol. 13, pp. 3993-4005, 1980.

[37] I. Thomann, et al., "Temporal characterization of attosecond wave forms in the sub-optical-cycle regime," Physical Review A, vol. 78, pp. 011806(R), 2008.

[38] E. A. Gibson, et al., "Extreme nonlinear optics: Attosecond photonics at short wavelengths," Ieee Journal of Selected Topics in Quantum Electronics, vol. 10, pp. 1339-1350, 2004.

[39] E. Gagnon, et al., "Time-resolved momentum imaging system for molecular dynamics studies using a tabletop ultrafast extremeultraviolet light source," Review of Scientific Instruments, vol. 79, pp. 063102, 2008.

[40] J. Ullrich, et al., "Recoil-ion and electron momentum spectroscopy: reaction-microscopes," Reports on Progress in Physics, vol. 66, pp. 1463-1545, 2003.

[41] A. T. J. B. Eppink and D. H. Parker, "Velocity map imaging of ions and electrons using electrostatic lenses: Application in photoelectron and photofragment ion imaging of molecular oxygen," Review of Scientific Instruments, vol. 68, pp. 3477-3484, 1997.

[42] L. G. Gouy, "Sur une propriete nouvelle des ondes lumineuses," C.R. Acad. Sci. Paris, vol. 110, pp. 1251, 1890.

[43] R. W. Boyd, "Intuitive Explanation of the Phase Anomaly of Focused Light-Beams," Journal of the Optical Society of America, vol. 70, pp. $877-880,1980$.

[44] S. M. Feng and H. G. Winful, "Physical origin of the Gouy phase shift," Optics Letters, vol. 26, pp. 485-487, 2001.

[45] A. Roberts, N. Shivaram, L. Xu, and A. Sandhu, "Optimization of few-cycle pulse generation: Spatial size, mode quality and focal volume effects in filamentation based pulse compression," Optics Express, vol. 17, pp. 23894-23902, 2009.

[46] N. Shivaram, H. Timmers, L. Xu, A. Roberts, and A. Sandhu, "Gouy Phase Gating of Two-color Ionization to Uncover Attosecond Structure," Oxford University Press (To be published), 2011.

[47] C. Buth, R. Santra, and L. Young, "Electromagnetically induced transparency for X rays," Physical Review Letters, vol. 98, pp. 253001, 2007.
[48] P. Ranitovic, et al., "IR-Assisted Ionization of Helium by Attosecond XUV Radiation," New Journal of Physics, vol. 12, pp. 013008, 2010.

[49] S. I. Chu and D. A. Telnov, "Beyond the Floquet theorem: generalized Floquet formalisms and quasienergy methods for atomic and molecular multiphoton processes in intense laser fields," Physics Reports-Review Section of Physics Letters, vol. 390, pp. 1-131, 2004.

[50] X. M. Tong and N. Toshima, "Controlling atomic structures and photoabsorption processes by an infrared laser," Physical Review A, vol. 81, pp. -, 2010.

[51] P. Riviere, O. Uhden, U. Saalmann, and J. M. Rost, "Strong field dynamics with ultrashort electron wave packet replicas," New Journal of Physics, vol. 11, pp. 053011, 2009.

[52] X. M. Tong, P. Ranitovic, C. L. Cocke, and N. Toshima, "Mechanisms of infrared-laser-assisted atomic ionization by attosecond pulses," Physical Review A, vol. 81, pp. 021404, 2010.

[53] M. Swoboda, et al., "Phase Measurement of Resonant Two-Photon Ionization in Helium," Physical Review Letters, vol. 104, pp. -, 2010 .

[54] X. M. Tong and N. Toshima, "Infrared-laser-assisted photoionization of helium by coherent extreme ultraviolet light," Physical Review A, vol. 81, pp. -, 2010.

[55] N. Shivaram, A. Roberts, L. Xu, and A. Sandhu, "In situ spatial mapping of Gouy phase slip for high-detail attosecond pump-probe measurements," Optics Letters, vol. 35, pp. 3312-3314, 2010.

[56] M. C. Chen, et al., "Bright, Coherent, Ultrafast Soft X-Ray Harmonics Spanning the Water Window from a Tabletop Light Source," Physical Review Letters, vol. 105, pp. -, 2010.

[57] T. Popmintchev, et al., "Phase matching of high harmonic generation in the soft and hard X-ray regions of the spectrum," Proceedings of the National Academy of Sciences of the United States of America, vol. 106, pp. 10516-10521, 2009.

[58] G. Doumy, et al., "Attosecond Synchronization of High-Order Harmonics from Midinfrared Drivers," Physical Review Letters, vol. 102, pp. 093002, 2009.

[59] R. Santra, J. Zobeley, L. S. Cederbaum, and N. Moiseyev, "Interatomic Coulombic decay in van der Waals clusters and impact of nuclear motion," Physical Review Letters, vol. 85, pp. 4490-4493, 2000.

[60] R. Santra, J. Zobeley, and L. S. Cederbaum, "Inner-valence ionization of molecular anions and ultrafast relaxation by electron emission," Chemical Physics Letters, vol. 324, pp. 416-422, 2000. 